\title{
Diagnostic value of microRNAs derived from exosomes in bronchoalveolar lavage fluid of early-stage lung adenocarcinoma: A pilot study
}

\author{
Ji Eun Kim ${ }^{1 *}$, Jung Seop Eom ${ }^{*}$, Won-young Kim ${ }^{1}$, Eun Jung Jo', Jeongha Mok ${ }^{1}$, Kwangha Lee', \\ Ki Uk Kim¹, Hye-kyung Park', Min Ki Lee ${ }^{1,2}$ \& Mi-hyun Kim ${ }^{1,2}$ (1) \\ 1 Department of Internal Medicine, School of Medicine, Pusan National University, Busan, Republic of Korea \\ 2 Medical Research Institute, Pusan National University, Busan, Republic of Korea
}

\section{Keywords \\ Diagnosis; exosome; lung adenocarcinoma; microRNA.}

\section{Correspondence \\ Mi-hyun Kim, Department of Internal \\ Medicine, School of Medicine, Pusan National \\ University, 179, Gudeok-ro, Seo-gu, Busan \\ 49241, Republic of Korea. \\ Tel: +82512407497 \\ Fax: +82 512543127 \\ Email: mihyunkim@pusan.ac.kr \\ *Ji Eun Kim and Jung Seop Eom equally contributed to this work.}

Received: 31 January 2018;

Accepted: 9 April 2018

doi: 10.1111/1759-7714.12756

Thoracic Cancer 9 (2018) 911-915

\begin{abstract}
Background: Low-dose computed tomography can identify smaller nodules more often than chest radiography in lung screening. However, complications from invasive diagnostic procedures performed to detect nodules are common. Exosomes contain a diverse array of biomolecules that reflect the biological state of the cell from which they are released. The aim of this study was to investigate the diagnostic value of bronchoalveolar lavage (BAL) fluid exosomal microRNAs (miRNAs) for early-stage lung adenocarcinoma.

Methods: We evaluated miRNAs (miR-7, miR-21, miR-126, Let-7a, miR-17, and miR-19) known to have diagnostic value for lung adenocarcinoma. Exosomes were isolated from the BAL fluid of control subjects $(n=15)$ and patients with lung adenocarcinoma $(n=13)$. Exosomal miRNA was analyzed using a commercial kit containing probes targeting six selected miRNAs. Results were validated via quantitative PCR.

Results: The presence of miRNAs was confirmed in exosomes from BAL fluid of both lung adenocarcinoma patients and control subjects. miR-126 $(P<0.001)$ and Let-7a $(P=0.015)$ levels were significantly higher in the BAL fluid of lung adenocarcinoma patients than in control subjects. The BAL fluid miRNA signature was confirmed using an independent set of paired adenocarcinoma and normal tissue samples $(n=4)$. Lung adenocarcinoma tissues showed increased expression of miR-126 $(P=0.039)$ compared to normal tissue samples.

Conclusion: We identified a close correlation between BAL fluid exosomal miRNAs and tumor miRNAs. BAL fluid exosomal miRNAs obtained through noninvasive methods could serve as diagnostic biomarkers in early-stage lung adenocarcinoma.
\end{abstract}

\section{Introduction}

Lung cancer is the leading cause of cancer-related death worldwide. Most patients with lung cancer are diagnosed at an advanced stage, and the current five-year survival rate is only $18.1 \%{ }^{1}$ However, the five-year survival rate in patients with resected stage I non-small cell lung cancer (NSCLC) can be as high as $83 \% .{ }^{1}$ This difference is a result of the principle that detecting tumors at a smaller size and earlier stage, when treatment is more effective, results in a reduction in lung cancer mortality. ${ }^{2,3}$ Lung adenocarcinoma is now the most common histologic type of NSCLC, accounting for $40 \%$ of all NSCLCs. ${ }^{1}$ Lung adenocarcinoma is difficult to detect by bronchoscopy or sputum cytology, because it usually arises in peripheral lung tissue and originates from the smaller airways. ${ }^{4}$

Based on recent results from the National Lung Screening Trial, low-dose computed tomography has been suggested as a screening tool for lung cancer. ${ }^{5}$ This trial demonstrated an encouraging 20\% relative reduction in lung cancer-specific mortality compared to screening by chest radiography. ${ }^{5}$ However, improved sensitivity is 
associated with several problems, for example, overdiagnosis and complications from invasive diagnostic procedures on detected nodules. Thus, development of an adequate method for early detection remains an important issue in the management of lung adenocarcinoma.

Exosomes, small membrane vesicles $30-100 \mathrm{~nm}$ in size, are actively released from multiple cell types, including dendritic cells, lymphocytes, and tumor cells by exocytosis. ${ }^{6}$ They mediate cell-to-cell communication by transferring proteins, lipids, and nucleic acids between cells, resulting in the transformation of wild-type cells into malignant cells. ${ }^{7,8}$ As a diagnostic cancer marker, exosomes have been evaluated in many malignant tumors, such as melanoma and ovary, prostate, and cervical cancers. ${ }^{9-14}$ Only a few small-scale studies have explored the potential of exosomes as diagnostic biomarkers in lung adenocarcinoma. ${ }^{15-17}$ Until now, membrane proteins such as CD91, CD317, and EGFR, as well as a panel of 12 microRNAs (miRNAs), have been shown to be potential exosomal markers of NSCLC. ${ }^{15-17}$

MiRNAs are non-coding RNA molecules approximately 22-23 nucleotides long that regulate gene expression in organisms ranging from nematodes to humans. ${ }^{18}$ In recent years, numerous oncogenic miRNAs associated with lung cancer tumorigenesis have been reported. ${ }^{19}$ The majority of miRNAs are intracellular, and only a small number has been detected extracellularly, including in various bodily fluids. ${ }^{20-22}$ Extracellular miRNAs are remarkably stable despite high extracellular RNase activity, ${ }^{22}$ suggesting that these miRNAs are protected from RNase digestion. Recent studies have confirmed that extracellular miRNAs are packaged in specific extracellular vesicles - exosomes - thereby shielding them from RNase degradation. ${ }^{7,21}$

Based on their biological functions and stability, exosomal miRNAs may be a promising class of potential noninvasive biomarkers for lung cancer. The objective of this pilot study was to investigate whether exosomal miRNAs detected in bronchoalveolar lavage (BAL) fluid provide a useful biomarker for diagnosis of early-stage lung adenocarcinoma.

\section{Methods}

\section{Patient samples}

We prospectively obtained BAL samples from 13 lung adenocarcinoma patients and 15 control patients with nontumor pathology (e.g. interstitial lung disease [ILD]), between December 2014 and 2015. Bronchoscopy was clinically indicated in these patients to complement the diagnostic process. Lung adenocarcinoma patients at pathological stage I or II according to the International
Association for the Study of Lung Cancer 7th Tumor Node Metastasis staging system were included in this study. The most frequent pathologies in patients with ILD were nonspecific interstitial pneumonia $(n=4)$, chronic eosinophilic pneumonia $(n=3)$, sarcoidosis $(n=2)$, cryptogenic organizing pneumonia $(n=2)$, hypersensitivity pneumonitis $(n=2)$, idiopathic pulmonary fibrosis $(n=1)$, and acute eosinophilic pneumonia $(n=1)$. BAL cells were pelleted by centrifugation at $400 \times g$ for 10 minutes at $4^{\circ} \mathrm{C}$, and the cell-free supernatant was stored at $-80^{\circ} \mathrm{C}$ prior to exosome isolation.

The study protocol was approved by the medical research committee of the College of Medicine and Health Sciences, and was conducted in accordance with the ethical guidelines of the 1975 Helsinki Declaration. Informed consent was obtained from all participants, as approved by the institutional review board of the Pusan National University Hospital (H-1411-005-023).

\section{Exosome isolation from bronchoalveolar lavage (BAL) fluid}

Exosomes were isolated from BAL fluid using a commercial kit according to manufacturer protocols (ExoQuick Exosome Precipitation Solution, System Biosciences, Palo Alto, CA, USA). In brief, BAL fluid was centrifuged at $3000 \times g$ for 15 minutes to remove cells and cell debris, and then the appropriate volume of precipitation solution was added to the BAL fluid, followed by centrifugation at $1500 \times g$ for 30 minutes. The pellet was stored at $-80^{\circ} \mathrm{C}$ for subsequent applications.

\section{MicroRNA (MiRNA) extraction}

Exosomal miRNAs were isolated using the mirVana miRNA Isolation Kit (Ambion, Austin, TX, USA) after RNase A (Qiagen GmbH, Hilden, Germany) digestion of miRNAs outside of the exosomes. Reverse transcription and quantitative PCR were performed using the MicroRNA TaqMan Reverse Transcription Kit and TaqMan MicroRNA Assay (Applied Biosystems, Foster City, CA, USA) to identify candidate miRNAs (miR-7, miR-21, miR-126, Let-7a, miR-17, and miR-19). Data were analyzed with MxPro-Mx3005P v.3.00 (Stratagene, La Jolla, CA, USA) with the automatic comparative threshold cycle $\left(C_{t}\right)$ setting for adapting baseline values and determining the $C_{t}$ threshold. Expression levels of mature miRNAs were normalized to that of U6 $\left(2^{-\Delta \mathrm{Ct}}\right)$, and the fold change in each miRNA was calculated from differences in expression level between lung adenocarcinoma and controls. 


\section{Statistical analysis}

Given the small sample sizes, miRNA levels were compared using the Mann-Whitney $U$ test. SPSS version 18.0 (SPSS Inc., Chicago, IL, USA) was used for statistical analyses. $P$ values $<0.05$ were considered statistically significant.

\section{Results}

\section{Characteristics of patients with lung adenocarcinoma}

The characteristics of the 13 patients enrolled in this study are summarized in Table 1 . The mean age of the patients was 62 years, and seven patients $(53.8 \%)$ were male. The most common location of the primary tumor was the lower lobe of the right lung $(n=6,46.2 \%)$. The mean size of the primary tumor was $2.7 \mathrm{~cm}$ (range $1.4-4.6 \mathrm{~cm}$ ).

\section{Differences in exosomal miRNA levels in the BAL fluid of lung adenocarcinoma patients and controls}

Based on previous reports, six miRNAs (miR-7, 17, 19, 21,126 , and Let-7a) were chosen for investigation in this study. ${ }^{23}$ These six miRNAs have demonstrated diagnostic value for lung adenocarcinoma. The six miRNAs were quantified from exosomes derived from the BAL fluid samples of 13 lung adenocarcinoma patients and 15 controls (patients with benign lung diseases). Exosomal miRNA$126(P<0.001)$ and Let-7a $(P=0.015)$ levels were significantly higher in the BAL fluid of lung adenocarcinoma patients than in control subjects (Fig 1). There were no

Table 1 Lung adenocarcinoma patient characteristics

\begin{tabular}{llllcc}
\hline Cases & Gender & Age & $\begin{array}{c}\text { Tumor } \\
\text { location }\end{array}$ & $\begin{array}{c}\text { Tumor } \\
\text { size }(\mathrm{cm})\end{array}$ & $\begin{array}{c}\text { Pathologic } \\
\text { stage }\end{array}$ \\
\hline 1 & Male & 70 & $\mathrm{RLL}$ & 4.5 & IB \\
2 & Male & 68 & $\mathrm{RLL}$ & 3.4 & IIA \\
3 & Female & 58 & $\mathrm{RLL}$ & 1.7 & IA \\
4 & Female & 57 & $\mathrm{RLL}$ & 2.9 & IA \\
5 & Female & 47 & $\mathrm{RLL}$ & 4.6 & IA \\
6 & Male & 72 & $\mathrm{LLL}$ & 1.8 & IA \\
7 & Male & 50 & $\mathrm{LUL}$ & 2.0 & IA \\
8 & Male & 69 & $\mathrm{RUL}$ & 1.4 & IA \\
9 & Female & 67 & $\mathrm{RLL}$ & 2.2 & IIA \\
10 & Female & 55 & $\mathrm{LUL}$ & 3.9 & IB \\
11 & Male & 72 & $\mathrm{RML}$ & 2.1 & IA \\
12 & Male & 58 & $\mathrm{RML}$ & 2.5 & IA \\
13 & Female & 67 & $\mathrm{RML}$ & 2.2 & IA \\
\hline
\end{tabular}

LLL, left lower lobe; LUL, left upper lobe; RLL, right lower lobe; RML, right middle lobe; RUL, right upper lobe. significant differences in other miRNA levels in BAL fluid between the two groups.

\section{Validation of BAL fluid exosomal miRNAs in tumor tissues}

These two miRNAs were validated in independent samples of paired lung adenocarcinoma and normal lung tissues $(n=4)$. MiRNA-126 was upregulated in the cancer samples relative to the corresponding normal lung tissues $(P=0.039)$; however, there was no difference in Let-7a level between the groups (Fig 2).

\section{Discussion}

The value of miRNAs present in tumor-derived exosomes has been intensively explored as a promising tool for diagnostic and prognostic methods in human cancers. ${ }^{24}$ Exosomes play an important role, not only as vehicles of intercellular communication, but also by transferring their contents to nearby cells. During this process, they specifically modulate the surrounding microenvironment, leading to tumor development and progression, thus affecting the therapeutic response. ${ }^{23}$

There has been intensive research into the role of miRNAs in exosomes in NSCLC, as very little is known to date. In 2009, Rabinowits et al. reported a significant difference in total exosome and miRNA levels between lung cancer patients and controls and found similarities between the patterns of circulating exosomal miRNAs and those of tumor-derived miRNAs. ${ }^{15}$ These results were the first to suggest that circulating exosomal miRNAs are useful as a screening test for lung adenocarcinoma. Many studies have used exosomal miRNAs isolated from plasma or serum, but few have examined exosomes in BAL fluid since the first study conducted by Admyre et al. ${ }^{25}$

Recently, exosomes isolated from BAL fluid in patients with lung neoplasms were investigated, but exosomal miRNAs were not included in that investigation. ${ }^{26}$ To our knowledge, no previous studies have investigated the diagnostic value of exosomal miRNAs from BAL fluid in early-stage lung cancer. In this prospective study, we demonstrated the presence of miRNAs in exosomes from the BAL fluid of both lung cancer patients and those with other lung diseases. We also showed that exosomal miRNAs, especially miR-126 and Let-7a, exhibited diagnostic value for early-stage lung adenocarcinoma. However, upregulation of Let-7a in BAL fluid was not confirmed in tumor tissues, which may have been a result of the small sample size.

MiR-126 has been shown to act as both a tumor suppressor and an oncogene, depending on the type of cancer. In general, miR-126 expression is decreased in lung cancer 
(a)

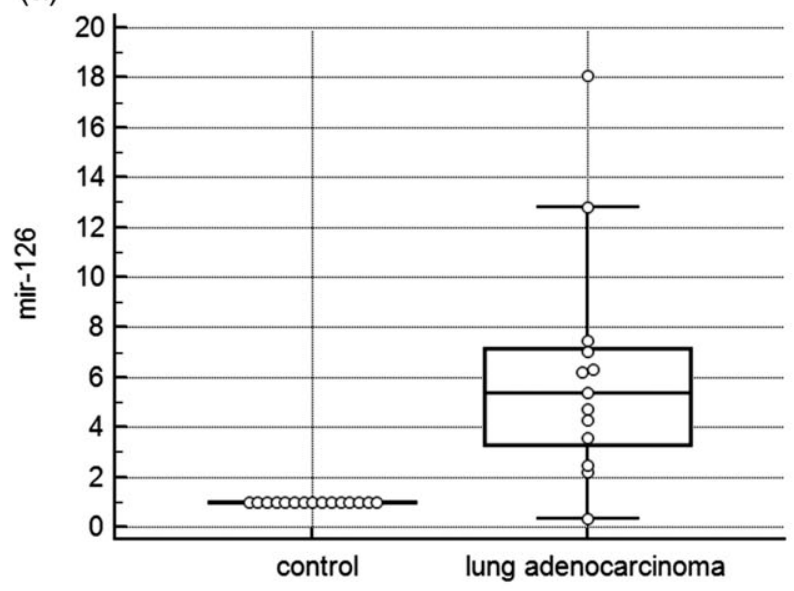

(b)

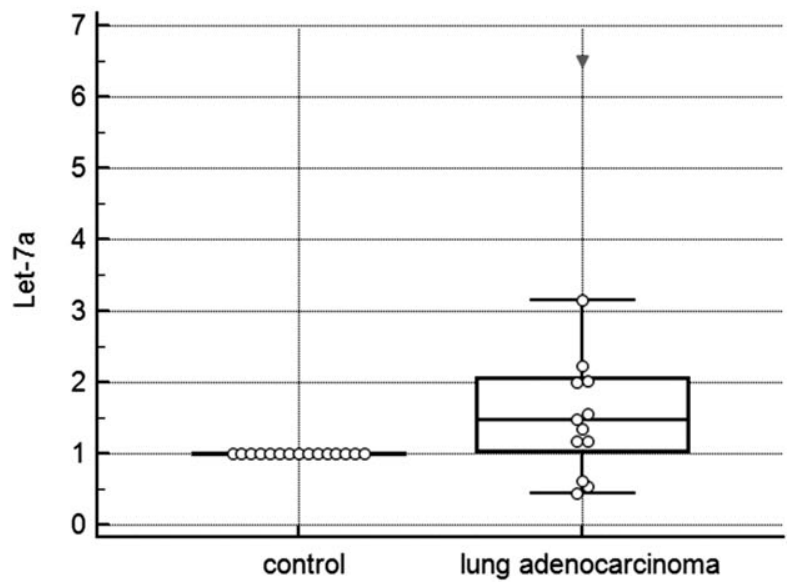

Figure 1 (a) Exosomal miRNA-126 ( $P<0.001)$ and (b) Let-7a $(P=0.015)$ levels in the bronchoalveolar lavage fluid of lung adenocarcinoma patients $(n=13)$ and control subjects $(n=15)$.

(a)

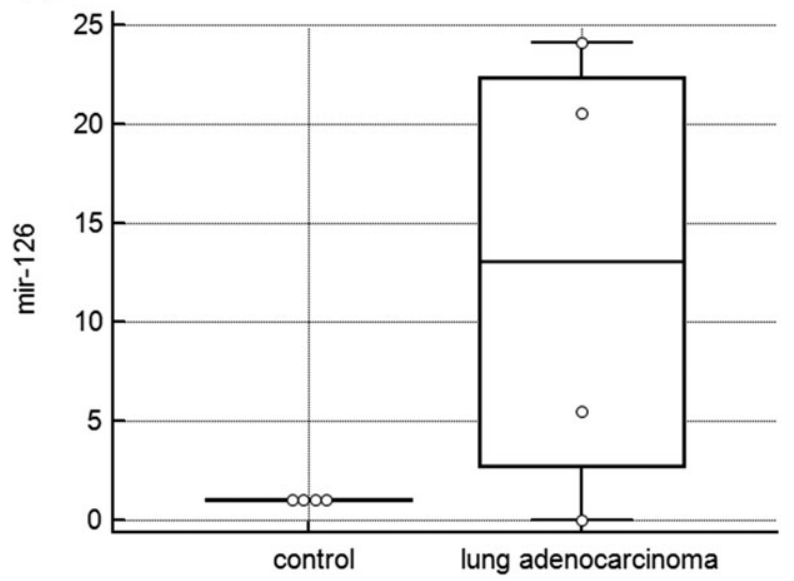

(b)

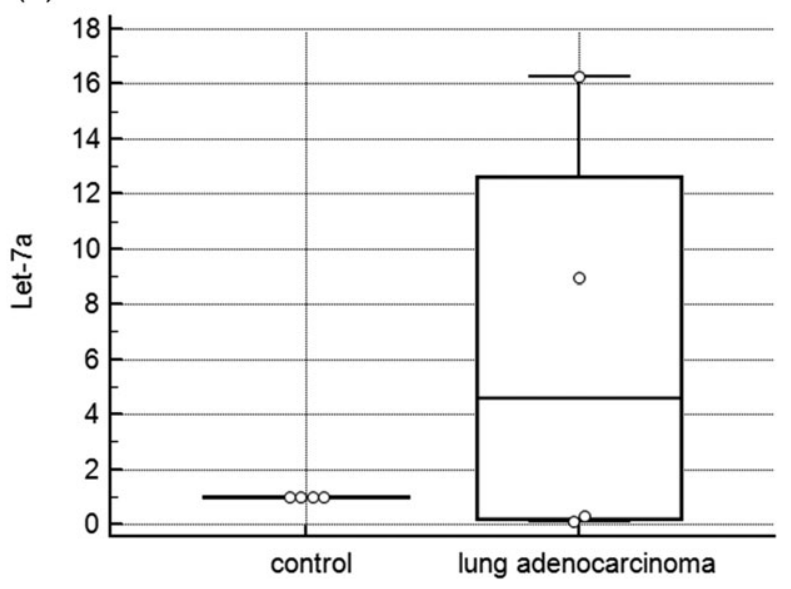

Figure 2 (a) Exosomal miRNA-126 (P=0.039) and (b) Let-7a $(P=0.258)$ levels in the samples of paired lung adenocarcinoma and normal lung tissues $(n=4)$.

cell lines, whereas increased expression of miR-126 inhibits proliferation of NSCLC cells in vitro and prevents tumor growth by targeting EGFL7. ${ }^{27}$ In this study, we examined exosomal miRNAs in BAL fluid from early-stage lung adenocarcinoma patients using a commercial kit. We identified a close correlation between BAL fluid exosomal miRNAs and tumor miRNAs. Thus, BAL fluid exosomal miRNAs obtained by noninvasive methods could serve as diagnostic biomarkers of early-stage lung adenocarcinoma.

There are some limitations to our study, including the small sample size. Because of the small sample, we could not determine whether there was an association between exosomal miRNA levels and clinical characteristics. Despite these limitations, our study confirmed the presence of miRNAs in exosomes from BAL fluid of both lung cancer patients and those with other lung diseases. Although the clinical feasibility of using exosomal miRNAs for lung cancer diagnosis was not confirmed, our results suggest the role of exosomal miRNAs in BAL fluid as a potential early diagnostic biomarker of lung cancer. These results need to be further investigated by larger, multi-center prospective studies. In addition, the role of exosomal miRNAs as biomarkers for predicting patient prognosis and response to chemotherapy should be investigated in long-term followup studies.

\section{Acknowledgments}

This study was supported by a Biomedical Research Institute Grant (2015-26) to the Pusan National University 
Hospital. The Biobank of Pusan National University Hospital, a member of the Korea Biobank Network, provided the biospecimens and data used for this study.

\section{Disclosure}

No authors report any conflict of interest.

\section{References}

1 Howlander N, Noone AM, Krapcho M et al. SEER Cancer Statistics Review. National Cancer Institute, Bethesda MD 1975-2014.

2 Patz EF Jr, Goodman PC, Bepler G. Screening for lung cancer. N Engl J Med 2000; 343: 1627-33.

3 Bach PB, Mirkin JN, Oliver TK et al. Benefits and harms of CT screening for lung cancer: A systematic review. (Published errata appear in JAMA 2013; 309: 2212; JAMA 2012; 308: 1324). JAMA 2012; 307: 2418-29.

4 Sun S, Schiller JH, Gazdar AF. Lung cancer in never smokers - A different disease. Nat Rev Cancer 2007; 7: 778-90.

5 National Lung Screening Trial Research Team, Aberle DR, Adams AM et al. Reduced lung-cancer mortality with lowdose computed tomographic screening. N Engl J Med 2011; 365: 395-409.

6 Simpson RJ, Lim JW, Moritz RL, Mathivanan S. Exosomes: Proteomic insights and diagnostic potential. Expert Rev Proteomics 2009; 6: 267-83.

7 Valadi H, Ekström K, Bossios A, Sjöstrand M, Lee JJ, Lötvall JO. Exosome-mediated transfer of mRNAs and microRNAs is a novel mechanism of genetic exchange between cells. Nat Cell Biol 2007; 9: 654-9.

8 Chen X, Liang H, Zhang J, Zen K, Zhang CY. Horizontal transfer of microRNAs: Molecular mechanisms and clinical applications. Protein Cell 2012; 3: 28-37.

9 Taylor DD, Gercel-Taylor C. MicroRNA signatures of tumor-derived exosomes as diagnostic biomarkers of ovarian cancer. (Published erratum appears in Gynecol Oncol 2010; 116: 153). Gynecol Oncol 2008; 110: 13-21.

10 Skog J, Würdinger T, van Rijn S et al. Glioblastoma microvesicles transport RNA and proteins that promote tumour growth and provide diagnostic biomarkers. Nat Cell Biol 2008; 10: 1470-6.

11 Logozzi M, De Milito A, Lugini L et al. High levels of exosomes expressing CD63 and caveolin-1 in plasma of melanoma patients. PLoS One 2009; 4: e5219.

12 Liu J, Sun H, Wang X et al. Increased exosomal microRNA21 and microRNA-146a levels in the cervicovaginal lavage specimens of patients with cervical cancer. Int J Mol Sci 2014; 15: 758-73.
13 Khan S, Jutzy JM, Valenzuela MM et al. Plasma derived exosomal survivin, a plausible biomarker for early detection of prostate cancer. PLoS One 2012; 7: e46737.

14 Gabriel K, Ingram A, Austin R et al. Regulation of the tumor suppressor PTEN through exosomes: A diagnostic potential for prostate cancer. PLoS One 2013; 8: e70047.

15 Rabinowits G, Gerçel-Taylor C, Day JM, Taylor DD, Kloecker GH. Exosomal microRNA: A diagnostic marker for lung cancer. Clin Lung Cancer 2009; 10: 42-6.

16 Li Y, Zhang Y, Qiu F, Qiu Z. Proteomic identification of exosomal LRG1: A potential urinary biomarker for detecting NSCLC. Electrophoresis 2011; 32: 1976-83.

17 Jakobsen KR, Paulsen BS, Bæk R, Varming K, Sorensen BS, Jørgensen MM. Exosomal proteins as potential diagnostic markers in advanced non-small cell lung carcinoma. J Extracell Vesicles 2015; 4: 26659.

18 Ambros V. MicroRNA pathways in flies and worms: Growth, death, fat, stress, and timing. (Published erratum appears in Cell 2003; 114: 269). Cell 2003; 113: 673-6.

19 Markou A, Zavridou M, Lianidou E. MicroRNA signatures as clinical biomarkers in lung cancer. Curr Biomarker Findings 2015; 5: 35-45.

20 Hunter MP, Ismail N, Zhang X et al. Detection of microRNA expression in human peripheral blood microvesicles. PLoS One 2008; 3: e3694.

21 Gilad S, Meiri E, Yogev Y et al. Serum microRNAs are promising novel biomarkers. PLoS One 2008; 3: e3148.

22 Mitchell PS, Parkin RK, Kroh EM et al. Circulating microRNAs as stable blood-based markers for cancer detection. Proc Natl Acad Sci U S A 2008; 105: 10513-8.

23 Sato-Kuwabara Y, Melo SA, Soares FA, Calin GA. The fusion of two worlds: Non-coding RNAs and extracellular vesicles - Diagnostic and therapeutic implications (review). Int J Oncol 2015; 46: 17-27.

24 Melo SA, Sugimoto H, O'Connell JT et al. Cancer exosomes perform cell-independent microRNA biogenesis and promote tumorigenesis. Cancer Cell 2014; 26: 707-21.

25 Admyre C, Grunewald J, Thyberg J et al. Exosomes with major histocompatibility complex class II and co-stimulatory molecules are present in human BAL fluid. Eur Respir $J$ 2003; 22: 578-83.

26 Rodríguez M, Silva J, López-Alfonso A et al. Different exosome cargo from plasma/bronchoalveolar lavage in nonsmall-cell lung cancer. Genes Chromosomes Cancer 2014; 53: 713-24.

27 Sun Y, Bai Y, Zhang F, Wang, Guo Y, Y, Guo L. miR-126 inhibits non-small cell lung cancer cells proliferation by targeting EGFL7. Biochem Biophys Res Commun 2010; 391 : 1483-9. 